\title{
AN ORDERED SET OF NÖRLUND MEANS
}

\author{
J. DEFRANZA \\ Department of Mathematics \\ St. Lawrence University \\ Canton, New York 13617 U.S.A.
}

(Received March 7, 1980 and in revised form Apri1 28, 1980)

\begin{abstract}
Norlund methods of summability are studied as mappings from $\ell_{1}$ into $\ell_{1}$. Those Norlund methods that map $s_{1}$ into $\ell_{1}$ are characterized. Inclusion results are given and a class of Norlund methods is shown to form an ordered abelian semigroup.

KEY WORDS AND PHRASES. Inclusion Theorem, $l-l$ method, ordered abelian semigroup, Norlund method.

1980 MATHEMATICS SUBJECT CLASSIFICATION CODES. Primary 40025, 40605.
\end{abstract}

1. INTRODUCTION.

Let $p$ be a complex sequence $p_{0} \neq 0$, and let $P_{n}=\sum_{k=0}^{n} p_{k}, n=0,1,2, \ldots$, denote the $n+1-s t$ partial sum of $p$. Suppose the sequence $P_{n}$ is eventually non-zero. Let $K$ be the least positive integer so that $P_{n} \neq 0$ for all $n \geqq K$. Define the Norlund method of summability $N_{p}$ by 


$$
N_{p}[n, k]=\left\{\begin{array}{l}
p_{n-k} / P_{0}, \text { if } 0 \leqq n<k, k \leqq n, \\
p_{n-k} / P_{n}, \text { if } n \geqq k, k \leqq n \text { and } \\
0, \text { otherwise. }
\end{array}\right.
$$

Let $N$ denote the collection of all such Norlund methods $N_{p}$. If $P_{n} \neq 0$ for all $\mathrm{n} \geqq 0$, that is $\mathrm{K}=0$, then

$$
N_{p}[n, k]=\left\{\begin{array}{cl}
P_{n-k} / P_{n}, & \text { if } k \leq n \text { and } \\
0, & \text { otherwise. }
\end{array}\right.
$$

Throughout we let $\hat{P}_{n}=P_{0}$ if $0 \leq n<K$ and $\hat{P}_{n}=P_{n}$ if $n \geqq K$. The $N_{p}$ transform of a sequence $x$ is then given by ${ }_{p} x$, where

$$
\left(N_{p} x\right)_{n}=\left(1 / \hat{p}_{n}\right) \sum_{k=0}^{n} p_{n-k} x_{k}
$$

for all $\mathrm{n} \geqq 0$.

A matrix sumnability method is called an $\ell-l$ method if and only if it maps the space $\varepsilon_{1} \equiv \ell$ Into Itself. In [4], Knopp and Lorentz proved that the matrix method $A$ is $\ell-\ell$ if and only if there exists some $M>0$ such that

$$
\sup _{k}\left\{\sum_{n=0}^{\infty}\left|a_{n k}\right|\right\}<M .
$$

In the special case of a Norlund method $N_{p}$ we have:

THEOREM 1. The Norlund method $N_{p}$ is $\ell-\ell$ if and only if

(1) $p \& \ell$, and

(11) $\hat{\mathrm{P}}_{\mathrm{n}}+0$ as $\mathrm{n} \rightarrow \infty$.

PROOF. First suppose that (1) and (11) hold. Since $p \varepsilon \ell$ it implies $\operatorname{Iim} \hat{P}_{n}$ exists and by (ii) is non-zero. So there exist strictly positive numbers 
$H$ and $\delta$ such that $\underset{n}{\Sigma}\left|p_{n}\right|<H$ and $\left|\hat{P}_{n}\right|>\delta$ for all $n \geqq 0$. Thus for each fixed $k$,

$$
\begin{aligned}
\sum_{n=k}^{\infty}\left|N_{p}[n, k]\right| & =\sum_{n=k}^{\infty}\left|p_{n-k} / \hat{p}_{n}\right| \\
& <(1 / \delta) \sum_{n=k}^{\infty}\left|p_{n-k}\right| \\
& <H / \delta .
\end{aligned}
$$

Thus by the Knopp-Lorentz Theorem $N_{p}$ is $\ell-\ell$.

Now suppose that $N_{p}$ is $\ell-\ell$. Then

$$
\sum_{n=k}^{\infty}\left|P_{n-k} / \hat{P}_{n}\right|=O(1)
$$

In particular if $n=k$ it implies $\left|1 / \hat{p}_{n}\right|=O(1)$ and hence $\hat{p}_{n}+0$ as $n \rightarrow \infty$.

Now suppose that $p \notin \ell$. We assert that the series $\underset{n}{\sum}\left(\left|p_{n} / \hat{P}_{n}\right|\right)$ diverges.

For sufficiently large $n$,

$$
\left|\hat{\mathrm{P}}_{\mathbf{n}}\right|=\left|\mathrm{P}_{\mathrm{n}}\right|=\left|\sum_{\mathbf{k}=0}^{\mathrm{n}} \mathrm{p}_{\mathbf{k}}\right| \leq \sum_{\mathrm{k}=0}^{\mathrm{n}}\left|\mathrm{p}_{\mathbf{k}}\right| \equiv \mathrm{S}_{\mathbf{n}} \text {. }
$$

Then for sufficiently large $N$

$$
\begin{aligned}
\sum_{n=N+1}^{N+m}\left(\left|p_{n} / \hat{P}_{n}\right|\right) & \geqq\left(1 / s_{N+m}\right) \sum_{n=N+1}^{N+m}\left|p_{n}\right| \\
& =1-s_{N} / S_{N+m} .
\end{aligned}
$$

But $\mathrm{S}_{\mathrm{N}+\mathrm{m}} \rightarrow \infty$ as $\mathrm{m} \rightarrow \infty$. So choose m large enough such that $\mathrm{S}_{\mathrm{N}+\mathrm{m}}>2 \mathrm{~S}_{\mathrm{N}}$.

Then $\sum_{n=N+1}^{N+m}\left(\left|p_{n}\right| /\left|\hat{P}_{n}\right|\right)>\frac{1}{2}$. The theorem now follows.

COROLLARY 1 [1, Theorem 4]. Let $\mathrm{N}_{\mathrm{p}}$ be a Norlund method with $\mathrm{p}_{\mathrm{n}} \geq 0, \mathrm{p}_{0}>0$. Then $N_{p}$ is $\ell-\ell$ if and only if $p \varepsilon l$.

2. We make the following definitions. 
DEFINITION. Let $N_{\ell}$ denote the collection of all $N_{p} \in N$ that are $\ell-\ell$ methods.

DEFINITION. Let $\ell\left(N_{p}\right)$ consist of all sequences $x$ such that $N_{p} x$ is in $\ell$. DEFinition. Given two Norlund methods $N_{p}$ and $N_{q}, N_{q}$ is $\ell$-stronger than $N_{p}$ if and only if $\ell\left(N_{p}\right) \subseteq \ell\left(N_{q}\right)$. The method $N_{q}$ is strictly $\ell$-stronger than $N_{p}$ provided $\ell\left(N_{p}\right) \subsetneq \ell\left(N_{q}\right)$, and $N_{p}$ and $N_{q}$ are $\ell$-equivalent provided $\ell\left(N_{p}\right)=\ell\left(N_{q}\right)$. DEFINITION. Given $N_{p}$ and $N_{q}$ define formally:

(1) $\mathrm{p}(\mathrm{z})=\sum_{\mathrm{n}=0}^{\infty} \mathrm{p}_{\mathrm{n}} \mathrm{z}^{\mathrm{n}}, \mathrm{P}(\mathrm{z})=\sum_{\mathrm{n}=0}^{\infty} \hat{\mathbf{P}}_{\mathrm{n}} z^{\mathrm{n}}$,

(i1) $q(z)=\sum_{n=0}^{\infty} q_{n} z^{n}, Q(z)=\sum_{n=0}^{\infty} \hat{Q}_{n} z^{n}$, and

(111) $a(z)=p(z) / q(z)=\sum_{n=0}^{\infty} a_{n} z^{n}, b(z)=q(z) / p(z)=\sum_{n=0}^{\infty} b_{n} z^{n}$.

The next propositions follow by an argument similar to the one used in Theorem 18 of [3] .

PROPOSITION 1. If $N_{p} \varepsilon N_{\ell}$, then the series $\sum_{n} p_{n} z^{n}$ and $\sum_{n} \hat{P}_{n} z^{n}$ converge for $|z|<1$.

PROPOSITION 2. If $N_{p}, N_{q} \in N_{l}$, then the series $a(z)=\sum_{n} a_{n} z^{n}$ and $b(z)=\sum_{n} b_{n} z^{n}$ have positive radil of convergence and, moreover

(1) $p_{n}=a_{n} q_{0}+\ldots+a_{0} q_{n}$,

(ii) $\hat{\mathrm{P}}_{\mathrm{n}}=a_{n} \hat{Q}_{0}+\ldots+a_{0} \hat{Q}_{n}$,

(iii) $q_{n}=b_{n} p_{0}+\ldots+b_{0} p_{n}$, and

(iv) $\hat{Q}_{n}=b_{n} \hat{\mathrm{P}}_{0}+\ldots+b_{0} \hat{\mathrm{P}}_{n}$.

PROPOSITION 3. Suppose $N_{p} \in N_{\ell}$ and the sequence $S=\left\{S_{n}\right\}$ is in $\ell\left(N_{p}\right)$. Then the series $S(z)=\sum_{n} S_{n} z^{n}$ has positive radius of convergence. 
PROOF. Let $h(z)=1 / p(z)$. By Proposition $1, p(z)$ defines an analytic function for $|z|<1$. Since $p_{0} \neq 0$, by the continuity of $p(z)$ there exists some $\alpha \varepsilon(0,1)$ such that $p(z) \neq 0$ for all $z \varepsilon(-\alpha, \alpha)$. Therefore $h(z)=\sum h_{n} z^{n}$ has positive radius of convergence. Now for $n \geqq 0$,

$$
\sum_{k=0}^{n}\left[h_{n-k} \hat{P}_{k}\left(N_{p} S\right)_{k}\right]=S_{n}
$$

Since $S \& \ell\left(N_{p}\right)$, it follows that the series $\sum_{k=0}^{\infty} P_{k}\left(N_{P} S\right)_{k} z^{k}$ converges for $|z|<1$. Therefore $\sum_{n} S_{n} z^{n}$ has positive radius of convergence since

$$
\sum_{n=0}^{\infty} S_{n} z^{n}=\{h(z)\}\left\{\sum_{k=0}^{\infty} \hat{P}_{k}\left(N_{p} S\right)_{k} z^{k}\right\}
$$

3. The symmetric product $g=p * q$ of the sequences $p$ and $q$ is defined by $g_{n}=p_{0} q_{n}+\ldots+p_{n} q_{0}$ for all $n \geqq 0$. Given Norlund methods $N_{p}$ and $N_{q}$ in $N$ we say $N_{g}=N_{p^{*} q}$ is the symmetric product of $N_{p}$ and $N_{q}$ provided $N_{g} \in N$.

In order to prove an inclusion result for two Norlund methods in $N_{\ell}$ we need the following lemma.

Lemma 1. Let the complex sequences $p$ and $q$ be given and define $r=p * q$. Suppose $N_{p}, N_{r} \in N$. Then in order that $\ell\left(N_{p}\right) \subseteq \ell\left(N_{r}\right)$ it is necessary and sufficient that there is some $M>0$, independent of $k$, such that

$$
\left|\hat{\mathrm{P}}_{\mathbf{k}}\right| \sum_{\mathbf{n}=\mathbf{k}}^{\infty}\left|\mathrm{q}_{\mathrm{n}-\mathrm{k}} / \hat{\mathrm{R}}_{\mathrm{n}}\right|<M .
$$

Proof. Let $x=\left\{x_{n}\right\}$ be any sequence. Then

$$
\left(N_{r} x\right)_{n}=\left(1 / \hat{R}_{n}\right)_{k=0}^{n}\left[q_{n-k} \hat{P}_{k}\left(N_{p} x\right)_{k}\right]
$$


Let $e_{n k}=q_{n-k} \hat{p}_{k} / \hat{R}_{n}$ if $k \leqq n$, and 0 if $k>n$. Then by the Knopp-Lorentz Theorem $\ell\left(N_{p}\right) \subseteq \ell\left(N_{r}\right)$ if and only if there exists some $M>0$ such that

$$
\sup _{\mathbf{k}}\left\{\sum_{\mathbf{n}=\mathbf{k}}^{\infty}\left|e_{\mathbf{n k}}\right|\right\}<M .
$$

That 18,

$$
\sup _{\mathbf{k}}\left\{\left|\hat{P}_{k}\right| \sum_{n=k}^{\infty}\left|q_{n-k} / \hat{R}_{n}\right|<M\right.
$$

The lemma can now be used to get the desired inclusion result.

Theorem 2. Suppose $N_{p}, N_{q} \in N_{\ell}$. The $\ell\left(N_{p}\right) \subseteq \ell\left(N_{q}\right)$ if and only if $b=\left\{b_{n}\right\} \varepsilon l$.

Proof. In the previous lemma replace the sequence $q$ with the sequence $b$ which implies $r=p *_{b}=q$. Then $\ell\left(N_{p}\right) \subseteq \ell\left(N_{r}\right)=\ell\left(N_{q}\right)$ if and only if there exists some $M>0$, independent of $k$, such that

$$
\left|\hat{P}_{k}\right| \sum_{n=k}^{\infty}\left|b_{n-k} / \hat{Q}_{n}\right|<M .
$$

But since $N_{p}, N_{q} \in N_{\ell},\left|\hat{P}_{k}\right|$ and $\left|\hat{Q}_{k}\right|$ respectively are bounded by two strictly positive constants. Thus $\left|\hat{P}_{k}\right| \sum_{n=k}^{\infty}\left|b_{n-k} / \hat{Q}_{n}\right|<M$, Independent of $k$, is equivalent to b $\varepsilon \ell$.

Corollary 2. Suppose $N_{p}, N_{q} \in N_{\ell}$. Then

(1) $\ell\left(\mathbb{N}_{p}\right)=\ell\left(\mathbb{N}_{q}\right)$ if and only if both $a=\left\{a_{n}\right\} \varepsilon \ell$ and $b=\left\{b_{n}\right\} \varepsilon \ell$

and

$$
\text { (11) } \ell\left(N_{p}\right) \oint_{\ddagger} l\left(N_{q}\right) \text { if and only if } a=\left\{a_{n}\right\} \notin \ell \text { and } b=\left\{b_{n}\right\} \varepsilon \ell \text {. }
$$

Corollary 3. Suppose $N_{p} \in N_{\ell}$ and $h(z)=1 / p(z)$. Then $\ell\left(N_{p}\right)=\ell$ if and only if $h \varepsilon l$. 
Proof. Let I be the identity matrix so that $\ell(I)=\ell$. Then $I(z)=\sum_{n=0}^{\infty} 1 z^{n}=1$; that is $1_{0}=1$ and $1_{n}=0$ for all $n \geq 1$. Therefore $a(z)=p(z) / I(z)=p(z)$ and $b(z)=I(z) / p(z)=h(z)$. The corollary now follows.

Example. The binary matrix $B=\left(b_{n k}\right)$ is given by $b_{n k}=1$, if $n=k=0,1 / 2$, If $k=n-1$ or $k=n, n>1$, and 0 otherwise. Thus $B$ is the $\ell-\ell$ Norlund method de-

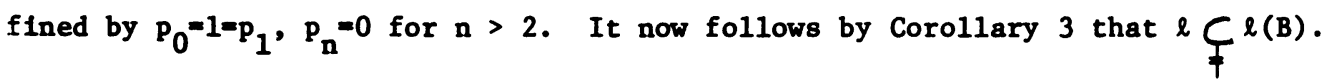

The next result addresses the question of when two Norlund methods $N_{p}, N_{q} \varepsilon N_{l}$ are comparable. We prove that changing only the first term in the generating sequence $N_{p}$ can result in a method $N_{q} \varepsilon N_{\ell}$ satisfying $\ell\left(N_{p}\right) \cap \ell\left(N_{q}\right)=\ell$. That 18 , the methods are not comparable.

Theorem 3. Suppose $N_{p} \in N_{\ell}$. Let $q=\left(p_{0}^{\prime}, p_{1}, \ldots\right)$ with $p_{0}^{\prime} \neq p_{0}$. If the sequence $q$ satisfies $\lim _{n \rightarrow \infty} \inf \left|Q_{n}\right| \neq 0$ then $N_{q}$ is $\ell-\ell$ and moreover $\ell\left(\mathrm{N}_{\mathrm{P}}\right) \cap \ell\left(\mathrm{N}_{\mathrm{q}}\right)=\ell$.

Proof. Since $q \in \ell, N_{q} \in N_{\ell},\left|\hat{P}_{n}\right|$ and $\left|\hat{Q}_{n}\right|$ respectively 11 e between two strictly positive constants. Now for any sequence $x$

$$
\left(N_{q} x\right)_{n}=\left(p_{0}^{\prime}-p_{0}\right) x_{n} / \hat{Q}_{n}+\left[\left(N_{p} x\right)_{n}\right]\left[\hat{P}_{n} / \hat{Q}_{n}\right],
$$

and hence

$$
\left|\left(\mathbb{N}_{q} x\right)_{n}\right|+\left|\hat{P}_{n} / \hat{Q}_{n}\right|\left|\left(\mathbb{N}_{p} x\right)_{n}\right| \geq\left|p_{0}^{\prime}-p_{0}\right|\left|x_{n}\right| /\left|\hat{Q}_{n}\right| \text {. }
$$

Therefore, if $x \in \ell\left(N_{p}\right), x \notin \ell$, then $x \notin \ell\left(N_{p}\right)$. Similarly if $x \in \ell\left(\mathbb{N}_{q}\right), x \notin \ell$, then $x \notin \ell\left(\mathbb{N}_{p}\right)$.

Corollary 4. Suppose $p$ is a positive number sequence in $\ell$. Let $q=\left(p_{0}^{\prime}, p_{1}, \ldots\right)$, where $p_{0}^{\prime}>0$ and $p_{0}^{\prime} \neq p_{0}$. Then $N_{p}$ and $N_{q}$ are $\ell-\ell$ with $\ell\left(\mathrm{N}_{\mathrm{p}}\right) \cap \ell\left(\mathrm{N}_{\mathrm{q}}\right)=\ell$. 
Theorem 4. Suppose $p$ is a sequence in $\ell$, with $P_{n}$ eventually non-zero. Let $q=\left(p_{1}, p_{2}, \ldots\right)$. If $Q_{n}$ is eventually non-zero, then $N_{p}, N_{q} \in N_{l}$ with $\ell\left(\mathrm{N}_{\mathrm{p}}\right) \cap \ell\left(\mathrm{N}_{\mathrm{q}}\right)=\ell$.

The next theorem is a special case of Theorem 2 .

Theorem 5. If $N_{p}$ and $N_{q}$ are Norlund methods with

(1) $p_{n+1} / p_{n} \geq p_{n} / p_{n-1}, n>0, p_{0}=1, p_{n}>0$,

(11) $q_{n} \geq 0, q_{0}=1$, and

(iii) $p, q \varepsilon \ell$,

then $\ell\left(N_{p}\right) \subseteq \ell\left(N_{q}\right)$.

Proof. By Theorem 22 of [3]

$\{p(z)\}^{-1}=1-c_{1} z-c_{2} z^{2}-\ldots$, where $c_{n}>0$ for all $n \geqq 0$ and $\sum_{n} \leqq 1$.

Then for small $|z|, q(z) / p(z)=\sum_{n=0}^{\infty}\left(q_{n} \gamma_{0}+\ldots+q_{0} \gamma_{n}\right) z^{n}$, where $\gamma_{n}=-c_{n}$ for $n>0$ and $\gamma_{0}=1$. So that if $q(z) / p(z)=\sum_{n=0}^{\infty} b_{n} z^{n}$, we have

$$
\begin{aligned}
\sum_{\mathbf{n}=0}^{\infty}\left|b_{n}\right| & \leq \sum_{n=0}^{\infty}\left(\left|q_{0}\right|\left|\gamma_{n}\right|+\ldots+\left|q_{n}\right|\left|\gamma_{0}\right|\right) \\
& =\left\{\sum_{n=0}^{\infty}\left|q_{n}\right|\right\}\left\{\sum_{n=0}^{\infty}\left|c_{n}\right|\right\} \\
& <\infty .
\end{aligned}
$$

Therefore by Theorem $2, \ell\left(N_{p}\right) \subseteq \ell\left(N_{q}\right)$.

Corollary 6. If $N_{p}$ and $N_{q}$ are $\ell-\ell$ Norlund methods such that

(1) $p_{n+1} / p_{n} \geq p_{n} / p_{n-1}, n>0, p_{0}=1, p_{n}>0$,

and

(11) $q_{n+1} / q_{n} \geqslant q_{n} / q_{n-1}, n>0, q_{0}=1, q_{n}>0$, then $\ell\left(N_{p}\right)=\ell\left(N_{q}\right)$. 
For example if $p_{n}=\rho^{n}$ and $q_{n}=\tau^{n}$ where $0<\rho, \tau<1$, then $\ell\left(N_{p}\right)=\ell\left(N_{q}\right)$. Moreover by Corollary $3, \ell\left(N_{p}\right)=\ell=\ell\left(N_{q}\right)$. If $\gamma_{n}=1 / n^{k}, k>1$ and $n>0$, then $\ell\left(\mathrm{N}_{\mathrm{p}}\right)=\ell\left(\mathrm{N}_{\gamma}\right)$.

4. We now show that $N_{\ell}$ forms an ordered abelian semigroup. The order relation is set inclusion between the absolute summability fields, and the binary operation is the symmetric product of the generating sequences. We need the following lemmas.

Lemma 2. Suppose $\mathrm{N}_{\mathrm{q}} \in N_{\ell}$ and $\mathrm{p}$ is a sequence for which $\lim _{\mathrm{n} \rightarrow \infty} \hat{\hat{\mathrm{P}}}_{\mathrm{n}}=\hat{\mathrm{P}} \neq 0$. Let $\mathrm{r}=\mathrm{p} * \mathrm{q} . \quad$ Then $\lim _{\mathrm{n} \rightarrow \infty}\left(\hat{\mathrm{R}}_{\mathrm{n}} / \hat{\mathrm{P}}_{\mathrm{Q}}\right)=1$.

Proof. We assert that $\mathrm{N}_{\mathrm{q}}$ is a regular method. By the Silverman-Toeplitz Theorem, see for example [6], it suffices to show that

(1) $q_{n-v} / \hat{Q}_{n} \rightarrow 0$ as $n \rightarrow \infty$ for each fixed $\nu \geqq 0$, and

(11) $\sum_{v=0}^{n}\left|q_{v}\right|=O\left(\left|\hat{Q}_{n}\right|\right)$.

But since $N_{q} \in N_{\ell},(i)$ and (ii) follow. Now for all n sufficiently large

$$
\hat{R}_{n} / \hat{Q}_{n}=\left(1 / \hat{Q}_{n}\right) \sum_{k=0}^{n} r_{k}=\left(1 / \hat{Q}_{n}\right) \sum_{k=0}^{n} q_{n-k} \hat{P}_{k} \text {, }
$$

which is the $N_{q}$ transform of the convergent sequence $\left\{P_{n}\right\}$. Thus $\lim _{n \rightarrow \infty}\left(\hat{R}_{n} / \hat{P Q}_{n}\right)=1$.

Lemma 3. If $N_{p}, N_{q} \varepsilon N_{\ell}$ and $r=p * q$, then $N_{r} \varepsilon N_{\ell}$.

Proof. By Lemma 1, we have $\lim _{n \rightarrow \infty} \hat{R}_{n}=\hat{P} \hat{Q} \neq 0$. In order to have $N_{r} \in N_{\ell}$, we need to show $N_{r}$ is an $\ell-\ell$ method. By Theorem 1 , it suffices to show that $r \varepsilon \ell$. But

$$
\begin{aligned}
\sum_{n=0}^{\infty}\left|r_{n}\right| & \leq\left\{\sum_{n=0}^{\infty}\left|p_{n}\right|\right\}\left\{\sum_{n=0}^{\infty}\left|q_{n}\right|\right\} . \\
& <\infty .
\end{aligned}
$$


Lemma 4. If $\mathrm{N}_{\mathrm{p}}, \mathrm{N}_{\mathrm{q}} \varepsilon N_{\ell}, \mathrm{r}=\mathrm{p} * \mathrm{q}$, then $\mathrm{N}_{\mathrm{r}}$ is $\ell-\ell$ with $\ell\left(\mathrm{N}_{\mathrm{p}}\right) \cup \ell\left(\mathrm{N}_{\mathrm{q}}\right) \subseteq \ell\left(\mathrm{N}_{\mathrm{r}}\right)$.

Proof. By Lemma 2, $N_{r} \in N_{\ell}$. By Theorem 2 it follows that $\ell\left(\mathrm{N}_{\mathrm{p}}\right) \cup \ell\left(\mathrm{N}_{\mathrm{q}}\right) \subseteq \ell\left(\mathrm{N}_{\mathrm{r}}\right)$

Lemma 5. Suppose $N_{p}, N_{q}, N_{s} \in N_{\ell}, \mu=p \star_{s}$, and $\nu=q *_{s}$.

(i) If $\ell\left(N_{p}\right) \subseteq \ell\left(N_{q}\right)$, then $\ell\left(N_{\mu}\right) \subseteq \ell\left(N_{\nu}\right)$.

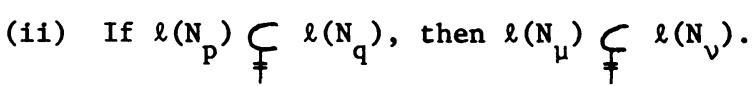

Proof of (i). Let $b(z)=q(z) / p(z)$ and $c(z)=v(z) / \mu(z)$. By Theorem 2, $\mathrm{b} \varepsilon \ell$. We need to show that $\mathrm{c} \varepsilon \ell$. Now for $|z|<1$,

$$
\begin{aligned}
v(z)=\sum_{n=0}^{\infty} v_{n} z^{n} & =\left\{\sum_{n=0}^{\infty} q_{n} z^{n}\right\}\left\{\sum_{n=0}^{\infty} s_{n} z^{n}\right\} \\
& =q(z) s(z) .
\end{aligned}
$$

Similarly $\mu(z)=p(z) s(z)$. Therefore

$$
\sum_{n=0}^{\infty} c n^{n}=c(z)=\nu(z) / \mu(z)=b(z)=\sum_{n=0}^{\infty} b_{n} z^{n} .
$$

Thus $\ell\left(\mathrm{N}_{\mu}\right) \subseteq \ell\left(\mathrm{N}_{\nu}\right)$.

The proof of (i1) follows by Theorem 2 and Corollary 2 .

A semigroup with order relation < is an ordered semigroup provided (i) $a<b$ and $b<c$ implies $a<c$, and (ii) $a<b$ implies $a c<b$ for $a l l c$. We now have the following theorem.

Theorem 6. With "strictly $\ell$-weaker than " as the order relation and * as the binary operation, $N_{\ell}$ is an ordered abelian semigroup.

Proposition 4. Let $N_{p} \in N_{\ell}$. Define $p_{-1}=0$ and $q_{n}=p_{n-1}+p_{n}$ for $n \geq 0$. If $Q_{n}$ is eventually non-zero, then $\ell\left(N_{p}\right) \oint_{f} \ell\left(N_{q}\right)$.

Proof. First note that $N_{q} \in N_{\ell}$. Moreover, it follows that $q(z)=(1+z) p(z)$ for $|z|<1$. Then by Corollary $2, \ell\left(N_{p}\right) \subseteq \ell\left(N_{q}\right)$. 
Proposition 5. There exists infinite chains of Nörlund methods from $N_{\ell}$.

Proof. Let $p^{(1)}(z)=\sum_{n=0}^{\infty} p_{n}{ }^{(1)} z^{n}$, where $\left\{p_{n}^{(1)}\right\} \varepsilon l, p_{n}^{(1)} \geqq 0$ for $n \geqq 0$, and $\mathrm{p}_{0}^{(1)}>0$. Then $\mathrm{N}_{\mathrm{p}}(1) \varepsilon N_{\ell}$. Define

$$
\mathrm{p}^{(\mathrm{n})}(\mathrm{z})=(1+\mathrm{z})^{\mathrm{n}-1} \sum_{\mathrm{k}=0}^{\infty} \mathrm{p}_{k}^{(\mathrm{n}-1)} \mathrm{z}^{\mathrm{k}} \text {, for } \mathrm{n} \geqq 2 \text {. }
$$

Then $N_{p}(n) \varepsilon N_{\ell}$ for $n \geqq 1$. Moreover by Proposition 4 we have

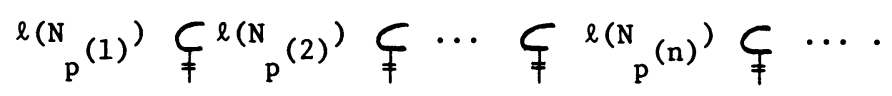

The next theorem is a different version of Lemma 3.

Theorem 7. If $N_{q} \in N_{\ell}, \lim _{n \rightarrow \infty} P_{n}=P \neq 0$, and $r=p * q$, then $\ell\left(N_{p}\right) \subseteq \ell\left(N_{r}\right)$.

Proof. By Lemma 1 it suffices to show there exists some $M>0$, Independent of $k$, such that

$$
\left|\hat{\mathrm{P}}_{\mathrm{k}}\right| \sum_{\mathrm{n}=\mathrm{k}}^{\infty}\left|\mathrm{q}_{\mathrm{n}-\mathrm{k}} / \hat{\mathrm{R}}_{\mathrm{n}}\right|<\mathrm{M} .
$$

Since $N_{q} \in N_{\ell}$, there exists some $\mathrm{H}>0$ such that

$$
\left|\hat{Q}_{n-k} / \hat{Q}_{n}\right|<H
$$

for all $n \geqq k$ and for all $k \geqq 0$. Then by Lemma 1 , we have

$$
\begin{aligned}
\left|\hat{P Q}_{n-k} / \hat{R}_{n}\right| & =\left|\hat{P} \hat{Q}_{n} / \hat{R}_{n}\right| \mid \hat{Q}_{n-k} / \hat{Q}_{n} \\
& <\infty
\end{aligned}
$$

for all $\mathrm{n} \geqq k$. Thus the result follows.

The author is indebted to the referee whose suggestions improved the exposition of these results. 


\section{REFERENCES}

1. J. A. Fridy, Absolute Summability Matrices That Are Stronger Than the Identity Mapping, Proc. Amer. Math. Soc., 47, I(1975), 112-118.

2. C. Goffman and H. V. Huneke, The Ordered Set of Nörlund Means, Math Z., $114(1970), 1-8$.

3. G. H. Hardy, Divergent Series, Oxford University Press (1949).

4. K. Knopp and G. G. Lorentz, Beiträge zur absoluten Limitierung, Arch. Math., 2(1949), 10-16.

5. B. Kwee, Some Theorems on Nörlund Summability, Proc. London Math. Soc. (3), $14(1964), 353-368$.

6. R. E. Powell and S. M. Shah, Summability Theory and Applications, Van Nostrand Reinhold Co., (1972).

7. B. Thorpe, On Inclusion Theorems and Consistency of Real Regular Nörlund Methods of Summability, J. London Math. Soc. (2), $\underline{5}(1972), 519-525$. 


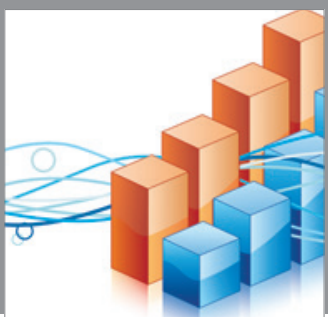

Advances in

Operations Research

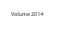

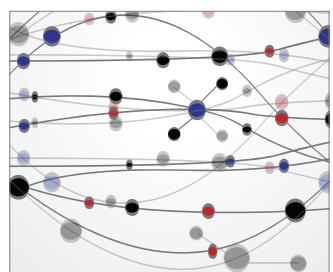

\section{The Scientific} World Journal
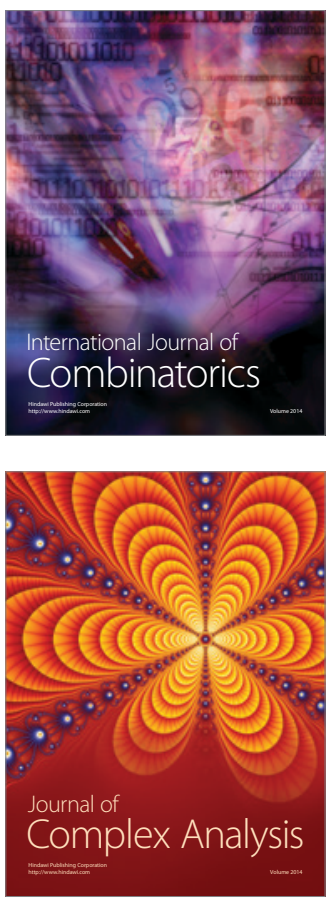

International Journal of

Mathematics and

Mathematical

Sciences
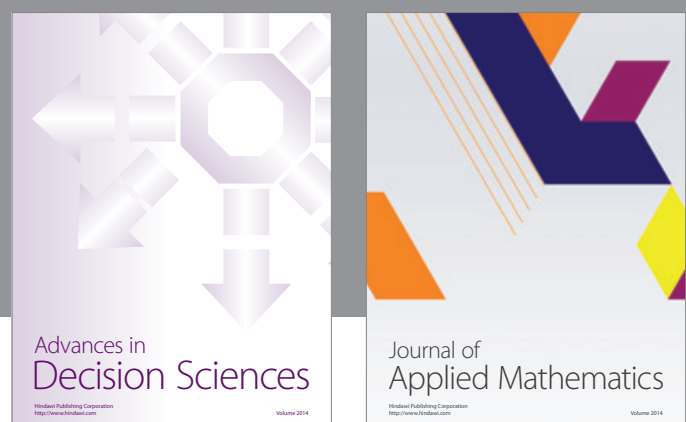

Journal of

Applied Mathematics
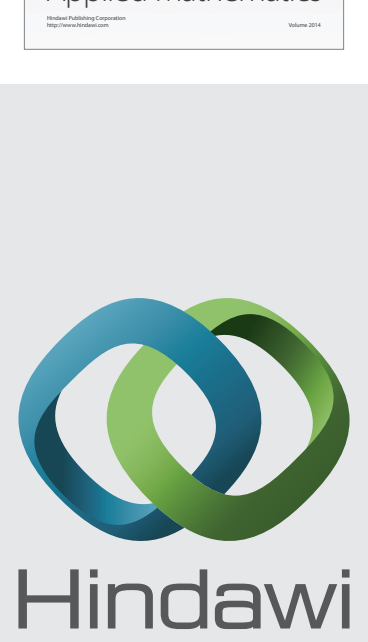

Submit your manuscripts at http://www.hindawi.com
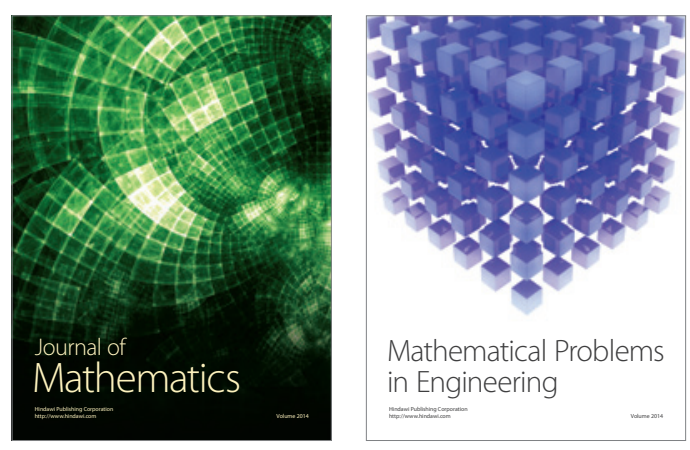

Mathematical Problems in Engineering
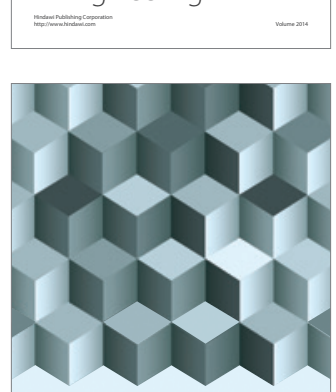

Journal of

Function Spaces
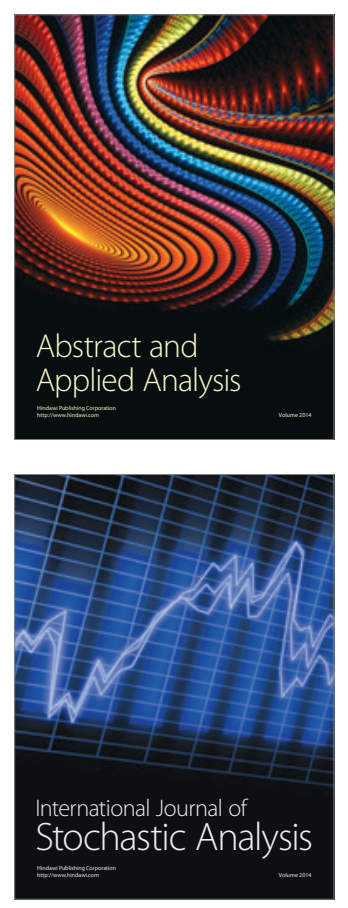

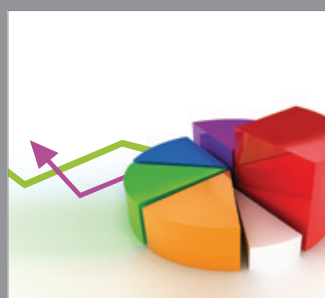

ournal of

Probability and Statistics

Promensencen
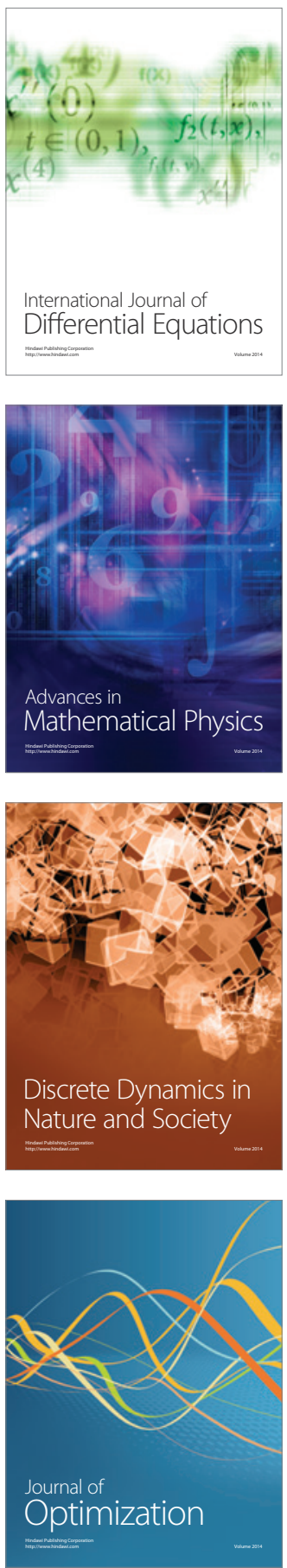